\title{
Comparación de inductores de la ovulación en un programa de IATF con semen sexado en novillas Bos taurus $x$ Bos indicus
}

\author{
Comparison of ovulation inducers in an FTAI programme with sexed semen \\ in Bos taurus $\mathbf{x}$ Bos indicus heifers
}
Diana Carolina Toro García ${ }^{1}$, Diego Armando Vega Borda ${ }^{1}$, Héctor Javier Narváez ${ }^{1.2}$, Deicy Villalba Rey ${ }^{1}$, Daniela Alvarado ${ }^{1}$

\section{Resumen}

\begin{abstract}
El objetivo del estudio fue comparar la eficiencia del cipionato de estradiol (ECP) y del benzoato de estradiol (BE) como inductores de la ovulación en un programa de inseminación artificial a tiempo fijo (IATF) utilizando semen sexado en novillas Senepol x Brahman rojo. Todos los animales recibieron en el día 0 un dispositivo intravaginal de progesterona ( $\mathrm{P} 4)$ de $0.5 \mathrm{~g}$ por ocho días $+2 \mathrm{mg}$ BE. A las novillas del grupo $1(\mathrm{n}=177) \mathrm{se}$ les aplicó $150 \mu \mathrm{g} \mathrm{PGF}_{2 \alpha}+150 \mathrm{UI}$ eCG $+1 \mathrm{mg}$ ECP y a las novillas del grupo $2(\mathrm{n}=177) 150$ $\mathrm{UI} \mathrm{eCG}+150 \mu \mathrm{g} \mathrm{PGF}_{2 \alpha}$ y $24 \mathrm{~h}$ más tarde recibieron $1 \mathrm{mg} \mathrm{BE}$. Las novillas fueron inseminadas con semen sexado $60 \mathrm{~h}$ pos-retiro del dispositivo de $\mathrm{P} 4$. Se determinó el diámetro del folículo preovulatorio y las tasas de gestación por tipo de inductor de ovulación. El diámetro del folículo preovulatorio en las novillas tratadas con ECP $(11.48 \pm 2.88 \mathrm{~mm})$ fue estadísticamente similar al de las tratadas con BE $(10.81 \pm 2.08 \mathrm{~mm})$. Se encontró una mayor tasa de gestación ( $\mathrm{p}=0.0069)$ en el grupo ECP $(51.4 \%)$ que en el grupo con BE (38.4\%). Se concluye que el CEP en comparación con el BE como inductor de la ovulación en protocolos de IATF favorece el crecimiento del folículo preovulatorio y las tasas de gestación.
\end{abstract}

Palabras clave: bovinos, IATF, tasas de gestación, dinámica folicular

\footnotetext{
${ }^{1}$ Universidad de Santander, Facultad de Ciencias Exactas, Naturales y Agropecuarias, Bucaramanga, Colombia

${ }^{2}$ E-mail: h.narvaez@mail.udes.edu.co
}

Recibido: 12 de marzo de 2021

Aceptado para publicación: 27 de septiembre de 2021

Publicado: 27 de octubre de 2021

CLos autores. Este artículo es publicado por la Rev Inv Vet Perú de la Facultad de Medicina Veterinaria, Universidad Nacional Mayor de San Marcos. Este es un artículo de acceso abierto, distribuido bajo los términos de la licencia Creative Commons Atribución 4.0 Internacional (CC BY 4.0) [https:// creativecommons.org/licenses/by/4.0/deed.es] que permite el uso, distribución y reproducción en cualquier medio, siempre que la obra original sea debidamente citada de su fuente original 
The aim of this study was to compare the efficiency of estradiol cypionate (ECP) and estradiol benzoate (EB) as ovulation inducers in a fixed-time artificial insemination programe (FTAI) using sexed semen in Senepol x Brahman red heifers. All animals received on day 0 an intravaginal device of progesterone (P4) of $0.5 \mathrm{~g}$ for eight days $+2 \mathrm{mg} \mathrm{BE}$. Heifersingroup $1(n=177)$ received $150 \mu \mathrm{g} \mathrm{PGF}_{2 \alpha}+150 \mathrm{IU}$ eCG $+1 \mathrm{mg}$ ECP and heifers in group $2(\mathrm{n}=177) 150 \mathrm{IU} \mathrm{eCG}+150 \mu \mathrm{g} \mathrm{PGF}_{2 \alpha}$ and $24 \mathrm{~h}$ later received $1 \mathrm{mg}$ BE. Heifers were inseminated with sexed semen $60 \mathrm{~h}$ after removal of the P4 device. The diameter of the preovulatory follicle and the gestation rates were determined by type of ovulation inducer. The diameter of the preovulatory follicle in heifers treated with ECP $(11.48 \pm 2.88 \mathrm{~mm})$ was statistically similar to those treated with $\mathrm{BE}(10.81 \pm 2.08 \mathrm{~mm})$. A higher gestation rate $(\mathrm{p}=0.0069)$ was found in the ECP group (51.4\%) than in the BE group (38.4\%). It is concluded that ECP in comparison with BE as an ovulation inducer in IATF protocols favours pre-ovulatory follicle growth and gestation rates.

Key words: bovine, FTAI, pregnancy rate, follicular dynamic

\section{INTRODUCCIÓN}

La inseminación artificial a tiempo fijo (IATF) es una biotecnología que ofrece grandes ventajas en comparación a la monta natural (Lamb y Mercadante, 2016) y tiene como objetivo el mejoramiento de la productividad y del potencial genético (Marizancén y Artunduaga, 2017; Baruselli et al., 2019). Así, la implementación de herramientas como la IATF en los programas de reproducción asistida permite avanzar en el mejoramiento genético y en la eficiencia reproductiva de los hatos, disminuyendo el intervalo entre partos e incrementando los índices de productividad (Pugliesi et al., 2017; Baruselli et al., 2019).

Los protocolos hormonales utilizados en los programas de IATF permiten concentrar la manifestación del estro y de la ovulación en un determinado espacio de tiempo (Peralta-Torres et al., 2010). La utilización de las sales de estradiol posterior al retiro del dispositivo de progesterona ( $\mathrm{P} 4)$ tiene como función inducir una retroalimentación positiva sobre la secreción de la hormona libera- dora de las gonadotrofinas ( $\mathrm{GnRH})$ y consecuentemente sobre la hormona luteinizante (LH) a fin de desencadenar la ovulación en un menor lapso (Cavalieri et al., 2002; Carvalho et al., 2017). En este sentido, los resultados obtenidos con el uso del Cipionato de estradiol y del Benzoato de estradiol como agentes promotores de la ovulación no han demostrado una diferencia significativa sobre las tasas de gestación en los protocolos utilizando semen convencional (Bó et al., 2019).

Por otro lado, la incorporación de semen sexado en los programas de IATF viene permitiendo una mejor selección de los animales, mayor producción de animales de reposición y mayor crecimiento del rebaño, además de una disminución de partos distócicos, debido a que el peso al nacimiento de las hembras es menor con relación al peso de los machos (Peres, 2014). Por esta razón, el objetivo del presente estudio fue comparar la eficiencia entre el cipionato de estradiol (ECP) y el benzoato de estradiol (BE) como inductores de la ovulación en un programa de IATF en novillas Bos taurus x Bos indicus utilizando semen sexado. 


\section{Materiales y Métodos}

\section{Animales y Diseño Experimental}

Se utilizaron 354 hembras bovinas de primer parto Senepol x Brahman Rojo (Bos taurus x Bos indicus) entre 24 y 26 meses de edad, $350 \mathrm{~kg}$ de peso vivo promedio y una condición corporal (CC) de 4.5 en la escala de 1 a 5 puntos, todas ellas pertenecientes a una ganadería local. Todos los animales fueron mantenidos en condiciones similares de pasturas a base de angleton (Dichanthium aristatum) y humidícola (Brachiaria humidicola), con suplementación mineral y agua ad libitum. Todas las hembras fueron vacunadas contra múltiples patógenos respiratorios y reproductivos (Vira Shield ${ }^{\circledR}$, Lab. Novartis, Suiza). El estudio fue realizado bajo condiciones tropicales con temperatura ambiental de $28{ }^{\circ} \mathrm{C}$ y precipitación promedio anual de $1160 \mathrm{~mm}$.

Para la sincronización del estro y de la ovulación, las hembras recibieron en el día 0 un dispositivo intravaginal de $\mathrm{P} 4$ de $0.5 \mathrm{~g}$ (Dispoce ${ }^{\circledR}$, Lab. Fatro Von Franken, Argentina) más la aplicación IM de $2 \mathrm{mg}$ de benzoato de estradiol (Syntex ${ }^{\circledR}$, Lab. Syntex, Argentina). El dispositivo se mantuvo in situ hasta el día 8 del protocolo, momento en que se evaluó el estado funcional de los ovarios por medio de ecografía (Pie Medical, Falco 100 con transductor de $7.5 \mathrm{MHz}$, Países Bajos) y la conformación de los grupos, tomando como criterio de inclusión la presencia del cuerpo lúteo (CL).

Ese mismo día (D8) se aplicó a las novillas del grupo $1(\mathrm{n}=177) 150 \mu \mathrm{g}$ de $\mathrm{PGF}_{2 \alpha}$ (Cloprostenol sódico - Sincrocio $^{\circledR}$, Lab. Ourofino, Brasil), 150 UI de eCG (Novormón $^{\circledR}$, Lab. Syntex, Argentina) y $1 \mathrm{mg}$ de ECP (Syntex ${ }^{\circledR}$, Lab. Syntex, Argentina). En forma similar, a las hembras del grupo 2 $(\mathrm{n}=177)$ se les aplicó una dosis de 150 UI de eCG IM (Novormón ${ }^{\circledR}$, Lab. Syntex, Argentina) y $150 \mu \mathrm{g}$ de $\mathrm{PGF}_{2 \alpha}$ y y $24 \mathrm{~h}$ más tarde se administró $1 \mathrm{mg}$ IM de BE (Syntex ${ }^{\circledR}$, Lab.
Syntex, Argentina) (D9). La IATF en ambos grupos fue realizada a las $60 \mathrm{~h}$ del retiro del dispositivo de progesterona utilizando semen sexado Gyr y Holstein (Sexed Ultra 4M ${ }^{\hat{a}}$ ) de una misma partida y de fertilidad reconocida. Todos los servicios de la inseminación artificial fueron realizados por el mismo técnico. Momentos antes de la IATF se evaluaron los ovarios para determinar el tamaño del folículo preovulatorio. El diagnóstico de la gestación fue realizado a los 45 días de la IATF mediante ecografía transrectal.

El análisis estadístico se realizó a través de una prueba de igualdad de medias (Franke et al., 2012), el cual utiliza la prueba de significancia de Chi-cuadrado.

\section{Resultados y Discusión}

La tasa de gestación global fue de $44.9 \%$ (159/354). El grupo de novillas tratadas con BE presentó una tasa de gestación del $38.4 \%$ $(68 / 177)$, mientras que en las hembras tratadas con ECP fue de $51.4 \%(91 / 177)(\mathrm{p}=0.007$; Figura 1). No obstante, algunos autores no encontraron diferencias significativas en porcentaje de concepción al utilizar ECP o BE como inductores de ovulación (Biondini et al.

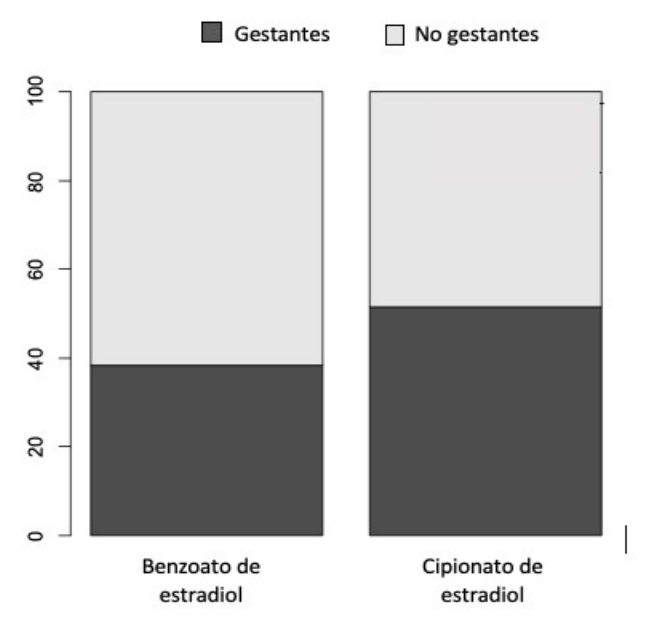

Figura 1. Porcentaje de novillas Bos taurus $\mathrm{x}$ Bos indicus que resultaron gestantes de acuerdo con el tipo de inductor de la ovulación utilizado (ECP, BE) en 


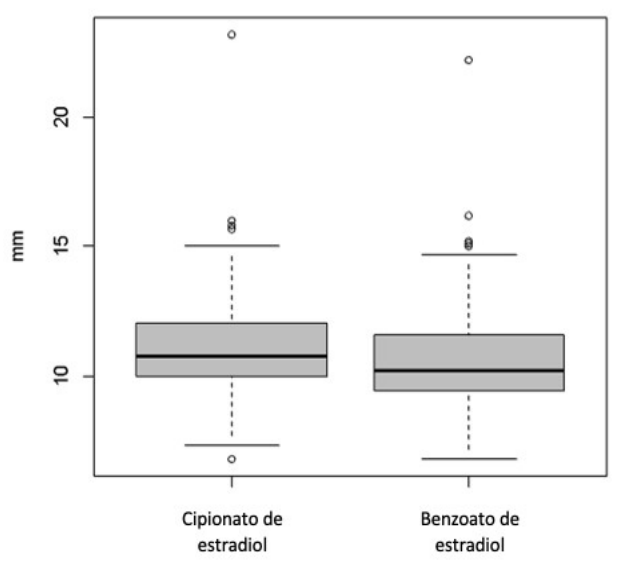

Figura 2. Efecto del benzoato de estradiol y del cipionato de estradiol en el tamaño del folículo preovulatorio en novillas Bos taurus $\mathrm{x}$ Bos indicus

[2011]: 52.2 vs. $53.4 \%$; respectivamente, con Bos taurus; Peralta Torres et al. [2010]: 72 vs. $79 \%$, respectivamente, con Bos indicus; Braz et al. [2013]: 65 y 57.4\% con Bos taurus; Crepaldi et al. [2019]: 63.3 vs. 66\%, con vacas Bos indicus).

Las divergencias encontradas entre los resultados del presente estudio con otros reportes pueden deberse a los múltiples factores que pueden afectar la fertilidad, tales como el estado nutricional (Calderón et al., 2017), el estado reproductivo (primíparas o multíparas) (Correa-Orozco y Velásquez, 2010), el estrés (Granja et al., 2012; Meneghetti et al., 2009), la presencia de la cría (Salazar, 1981) y la temperatura medio ambiental (Arias et al., 2008). Por otra parte, de Araújo Andrade et al. (2012) demostraron que los niveles circulantes de BE varían entre 1 y 23 horas y para el ECP entre 13 y 31 horas, lo que conlleva a una alta dispersión en la ovulación, afectando notablemente los resultados gestacionales.

El diámetro del folículo preovulatorio al utilizar ECP fue de $11.48 \pm 2.88$ y con BE fue de $10.81 \pm 2.08 \mathrm{~mm}$ (Figura 2), sin haber diferencias significativas entre ellos. Resultados similares fueron obtenidos por Bridges et al. (2013) en hembras Bos taurus y por Meneghetti et al. (2009) y Sá Filho et al. (2010) en vacas Bos indicus. Un estudio conducido por Gimenes et al. (2008), evidenciaron respuestas ovulatorias de 33,80 y $90 \%$ para folículos de 7.0-8.4, 8.5-10.0 y >10 mm, respectivamente.

Se reconoce que la fertilidad con semen sexado es inferior a la que se obtiene con semen convencional (Bó et al., 2019), debido a que el proceso de sexaje causa debilitamiento de los espermatozoides (Escapil et al., 2017); no obstante, las tasas de gestación obtenidas en el presente estudio fueron aceptables y comparables con las que se puede obtener con semen convencional.

\section{Conclusiones}

El uso del cipionato de estradiol en comparación con el benzoato de estradiol como inductor de la ovulación en protocolos de IATF favorece el crecimiento del folículo preovulatorio y las tasas de gestación.

\section{Agradecimentos}

Los autores agradecen a la Hacienda Medios Caños (Aguachica, Cesar, Colombia) por las facilidades prestadas para el desarrollo de la presente investigación.

\section{Literatura Citada}

1. Arias RA, Mader TL, Escobar PC. 2008. Factores climáticos que afectan el desempeño productivo del ganado bovino de carne y leche. Arch Med Vet 40: 7-22. doi: 10.4067/S0301-732X2008000100002

2. Baruselli P, Catussi BL, Abreu LÂ, Elliff F, Silva LG, Batista E, Crepaldi G. 2019. Evolução e perspectivas da inseminação artificial em bovinos. Rev Bras Reprod Anim 43: 308-314. 
3. Biondini M, Zangrilli G, Preisseger G, Gallejas S. 2011. Efectos de la sal de estradiol y de la duración del tratamiento con progesterona sobre el porcentaje de preñez a la IATF. Revista Veterinaria 22: $127-130$.

4. Bó GA, Huguenine E, De la Mata JJ, de Carneiro RJS, Menchaca A. 2019. Programas de IATF de alta fertilidad con semen sexado. En: XII Simposio Internacional de Reproducción Animal-IRAC. Argentina.

5. Braz M, Chayer R, Callejas S. 2013. Efecto de dos tratamientos de sincronización de la ovulación y el grado de desarrollo reproductivo sobre la eficiencia reproductiva de vaquillonas con 15 meses IATF. Rev. Taurus. https://www.researchgate.net/profile/Santiago_Callejas/publication/287193160_Effect_of_two_treatments_of_synchronization_of_ovulation_and three_levels_of_reproductive tract_scoring_on_pregnancy_rate _of_15_months_heifers_inseminated_at_a_fixed_time/links/56c4a99e08ae736e7046fa40.pdf

6. Calderón MF, Bosa LF, Yasnó JD, Saldaña LY. 2017. Relación nutriciónfertilidad en hembras bovinas en clima tropical. REDVET 18(9). [Internet]. Disponible en: https://www.redalyc.org/ pdf/636/63653009019.pdf

7. Carvalho NAT, Soares JG, Souza DC, Maio JRG, Sales JNS, Martins Júnior B, Macari RC, et al. 2017. Ovulation synchronization with estradiol benzoate or GnRH in a timed artificial insemination protocol in buffalo cows and heifers during the nonbreeding season. Theriogenology 87: 333-338. doi: 10.1016/ j.theriogenology.2016.09.006

8. Cavalieri J, Coleman C, Rodrigues $\mathrm{H}$, Macmillan KL, Fitzpatrick LA. 2002. The effect of timing of administration of oestradiol benzoate on characteristics of oestrus, timing of ovulation and fertility in Bos indicus heifers synchronised with a progesterone releasing intravaginal insert. Aust Vet J 80: 217-223. doi: 10.1111/j.1751-0813.2002.tb10817.x

9. Correa-Orozco A, Velásquez LFU. 2010. La condición corporal como herramienta para pronosticar el potencial reproductivo en hembras bovinas de carne. Rev Fac Nac Agron Medellín 63: 5607-5619.

10. Crepaldi GA, Sales JNS, Girotto RW, Carvalho JGS, Baruselli PS. 2019. Effect of induction of ovulation with estradiol benzoate at $\mathrm{P} 4$ device removal on ovulation rate and fertility in Bos indicus cows submitted to a TAI protocol. Anim Reprod Sci 209: 106141. doi: 10.1016/j.anireprosci.2019.106141

11. de Araújo Andrade BH, Ferraz PA, Rodrigues AS, Loiola MV, Chalhoub M, de Lisboa Ribeiro Filho A. 2012. Eficiência do cipionato de estradiol e do benzoato de estradiol em protocolos de indução da ovulação sobre a dinâmica ovariana e taxa de concepção de fêmeas nelore inseminadas em diferentes momentos. Arch Vet Sci 17: 70-82. doi: 10.5380/avs.v17i4.25710

12. Escapil JM, Cutaia L, Perez Wallace SM. 2017. Efecto de la utilización de cipionato de estradiol y detección de celos con pintura o benzoato de estradiol sobre la tasa de preñez a la IATF en vaquillonas Holando Argentino inseminadas con semen sexado. En; XII Simposio Internacional de Reproduccion Animal - IRAC. Argentina.

13. Franke TM, Ho T, Christie CA. 2012. The chi-square test: often used and more often misinterpreted. Am J Eval 33: 448458. doi: $10.1177 / 1098214011426594$

14. Bridges GA, Mussard ML, Helser LA, Day ML. 2014. Comparison of follicular dynamics and hormone concentrations between the 7-day and 5-day CO-Synch + CIDR program in primiparous beef cows. Theriogenology 81: 632-638. doi: 10.1016/j.theriogenology.2013.11.020 
15. Gimenes LU, Sá Filho MF, Carvalho NA, Torres-Júnior JR, Souza AH, Madureira EH, Trinca LA, et al. 2008. Follicle deviation and ovulatory capacity in Bos indicus heifers. Theriogenology, 69: 852-858. doi: 10.1016/ j.theriogenology.2008.01.001

16. Granja SY, Cerquera GJ, Fernandez BO. 2012. Factores nutricionales que interfieren en el desempeño reproductivo de la hembra bovina. Rev Colomb Cienc Anim 4: 458. doi: 10.24188/recia.v4.n2.2012.227

17. Lamb GC, Mercadante VR. 2016. Synchronization and artificial insemination strategies in beef cattle. Vet Clin N Am-Small 32: 335-347. doi: 10.1016/ j.cvfa.2016.01.006

18. Marizancén M, Artunduaga L. 2017. Mejoramiento genético en bovinos a través de la inseminación artificial y la inseminación artificial a tiempo fijo. Rev Inv Agraria Amb 8: 247-259. doi: $10.22490 / 21456453.2050$

19. Meneghetti M, Sá Filho OG, Peres RF, Lamb GC, Vasconcelos JL. 2009. Fixed-time artificial insemination with estradiol and progesterone for Bos indicus cows I: basis for development of protocols. Theriogenology 72: 179189. doi: $10.1016 /$ j.theriogenology.2009.02.010
20. Peralta-Torres J, Aké-López J, Centurión-Castro F, MaganaMonforte J. 2010. Comparison of estra-diol cypionate and estradiol benzoate effects on ovarian activity, estrus and ovulation on anestrus Bos indicus cows. J Anim Vet Adv 9: 466-470. doi: 10.3923/ javaa.2010.466.470

21. Pugliesi G, Rezende RG, Silva JD, Lopes E, Nishimura TK, Baruselli PS, Binelli M. 2017. Uso da ultrassonografia Doppler em programas de IATF e TETF em bovinos. Rev Bras Reprod Anim 41: 140-150.

22. Peres $A R$. 2014. Avaliação da taxa de concepção de novilhas e vacas (Bos taurus x Bos indicus) com o uso de sêmen sexado na inseminação artificial ou embriões produzidos in vivo e in vitro. Tesis de Maestría. Jaboticabal, Brasil: Univ. Estadual Paulista. 26 p.

23. Sá Filho MF, Ayres H, Ferreira RM, Marques MO, Reis EL, Silva RC, Rodrigues CA, et al. 2010. Equine chorionic gonadotropin and gonadotropin-releasing hormone enhance fertility in a norgestomet-based, timed artificial insemination protocol in suckled Nelore (Bos indicus) cows. Theriogenology 73: 651-658. doi: 10.1016/j.theriogenology.2009 .11 .004

24. Salazar JJ, Gallego MI, Gómez G, Rodríguez G. 1981. El problema de la reproducción bovina en Colombia. Instituto Colombiano Agropecuario. 